\title{
SURFACE-ENHANCED RAMAN SCATTERING: METAL NANOSTRUCTURES COATED WITH LANGMUIR-BLODGETT FILMS
}

\author{
${ }^{1}$ PRISCILA ALESSIO, ${ }^{1}$ CARLOS J. L. CONSTANTINO, ${ }^{2 \Delta}$ RICARDO F. AROCA, ${ }^{2 *}$ OSVALDO N. OLIVEIRA JR. \\ ${ }^{I}$ Faculdade de Ciências e Tecnologia, UNESP Univ Estadual Paulista, Presidente Prudente, 19060-080, SP, Brazil. \\ ${ }^{2}$ Instituto de Física de São Carlos, USP Universidade de São Paulo, São Carlos, SP, 13560-970, Brazil. \\ On leave from the department of Chem. and biochem, university of windsor, windsor, ontario, Canada. \\ (Received: August 30, 2010 - Accepted: November 16, 2010)
}

\begin{abstract}
This review deals with surface-enhancved Raman scattering (SERS) employing Langmuir-Blodgett (LB) films, which serve as model systems for developing theoretical and experimental studies to elucidate the SERS effect. In addition, LB films have be used as integral parts of molecular architectures for SERS-active substrates. On the other hand, SERS and surface-enhaced resonant Raman scattering (SERRS) have allowed various properties of LB films to be investigated, especially those associated with molecular-level interactions. In the paper, emphasis is placed on single molecule detection (SMD), where the target molecule is diluted on an LB matrix of spectral silent material (low Raman cross section). The perspectives and challenges for combining SERS and LB films are also discussed.
\end{abstract}

Keywords: Surface-enhanced Raman scattering, plasmonics, Langmuir-Blodgett films, nanoparticles.

\section{INTRODUCTION}

Metal nanoparticles ${ }^{1-3}$ have been extensively investigated due to their catalytic $^{4}$, biological ${ }^{5}$ and sensing ${ }^{6}$ properties, quite different from those in the bulk $^{7}$. In particular, $\mathrm{Ag}$ and $\mathrm{Au}$ nanostructures can sustain localized surface plasmon resonances (LSPR) in the visible range of the electromagnetic spectrum providing the basis for plasmon-enhanced spectroscopy that started with surface-enhanced Raman scattering (SERS) ${ }^{8,9}$. The local field enhancement is most efficient for the enhancement of the optical signals in aggregated nanoparticles (or similar structures) that can increase the spontaneous Raman scattering cross sections (of the order of $10^{-29} \mathrm{~cm}^{2}$ per molecule) ${ }^{10}$ by several orders of magnitude. An enhanced Raman signal was first reported in 1974 by Fleischmann et al..$^{11}$ with pyridine on rough Ag electrodes, an effect that was interpreted in 1977 as being due to a new physical phenomenon (SERS) by Jeanmaire and Van Duyne ${ }^{9}$ and Albrecht and Creighton ${ }^{8}$ in independent studies. The SERS effect is assigned to the enhancement of the optical signal when the incoming light can enable LSPR of the metal nanoparticles ${ }^{12,13}$. Basically, the electromagnetic enhancement mechanism (EM), at a given excitation frequency, depends on the dielectric function of the metal nanoparticles (mainly $\mathrm{Ag}$ and $\mathrm{Au}$ ), their shape, size and packing in the nanostructures ${ }^{14,15}$. It is also affected by the dielectric constant of the surrounding medium that wraps the metal nanoparticles. The intimate relationship between the plasmon excitation and the optical enhancement led to the development of plasmonics ${ }^{16}$. In many cases, the spectral properties of the species adsorbed onto the metal nanoparticles may change on account of the "chemical or physical" interactions with the nanostructure, and, correspondingly, the electromagnetically enhanced SERS spectrum will contain the information that shed light on these moleculenanostructure interactions $\mathbf{S}^{17,18}$.

The average SERS enhancement factor normally lies between $10^{3}$ and $10^{6}$, but the intense electromagnetic field in the interstices of the metal nanoparticles ("hot spots") can lead to a factor of up to $10^{10} 10,19$. Upon exploiting such hot spots and the high sensitivity of modern Raman spectrometers, it is possible to achieve single molecule detection (SMD) using SERS or surface-enhanced resonance Raman scattering (SERRS), which is the ultimate limit in terms of chemical analysis ${ }^{20-23}$. Since the highest enhancement factors are obtained with aggregates or clusters of nanoparticles (where hot spots are generated), there is a hefty number studies on the relationship of packing, size and shape of nanoparticles and the SERS signal ${ }^{19,24-28}$. The reproducible fabrication of nanostructures with maximum enhancement factor, as required for SMD, remains a challenge though. Different strategies have been developed to produce average enhancement factor (up to $10^{7}$ ), or higher, in SERS-active substrates, which involve traditional methods such as colloids ${ }^{29}$, large aggregated nanoparticles ${ }^{30,31}$, thermally evaporated thin $\operatorname{films}^{32}$, oblique angle vapor deposition ${ }^{33}$ and electrodeposition ${ }^{34}$, self-assembly of AuNPs with different diameters in an array ${ }^{35}$, dendrimer/Ag nanowire layer-by-layer (LbL) films $\mathrm{s}^{36}$, AgNP film formed by in-situ reaction ${ }^{37}$ or cast in a bottom-up strategy onto treated glass slides ${ }^{38}$, AuNPs distributed along nanocanals ${ }^{39}$, polymer-encapsulated AuNPs by self-assembly ${ }^{40}$ and gap-tailorable $\mathrm{Au}-\mathrm{Ag}$ core-shell nanodumbbells ${ }^{41}$. Recently, we reported on LbL films containing AgNPs trapped within a phospholipid matrix ${ }^{42}$ to achieve SERS and SERRS, which can be used in investigating interactions between guest molecules and phospholipids in membrane mimetic systems. There have also been reviews on the various methods to prepare $\mathrm{Ag}$ or $\mathrm{Au}$ SERS substrates for analytical applications, as in Lin et al. ${ }^{43}$. Two possibilities for obtaining SERS-substrates are illustrated in Figures 1 and 2.
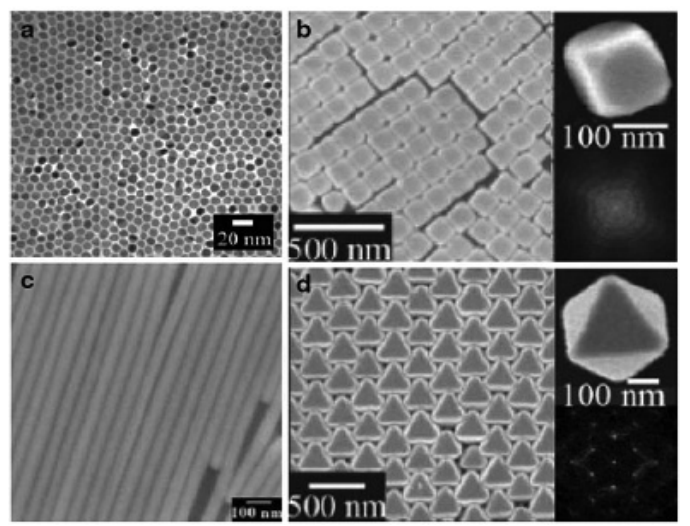

Figure 1: SEM images of a monolayer of nanocrystal and nanowires prepared with the Langmuir-Blodgett technique: a) Pt nanospheres, b) Ag nanocubes, c) Ag nanowires, d) Ag octahedral nanoparticles. Reproduced with permission from: X.-M. Lin, Y. Cui, Y.-H. Xu, B. Ren, Z.-Q. Tian. Analytical and Bioanalytical Chemistry 394, 1729-1745 (2009).

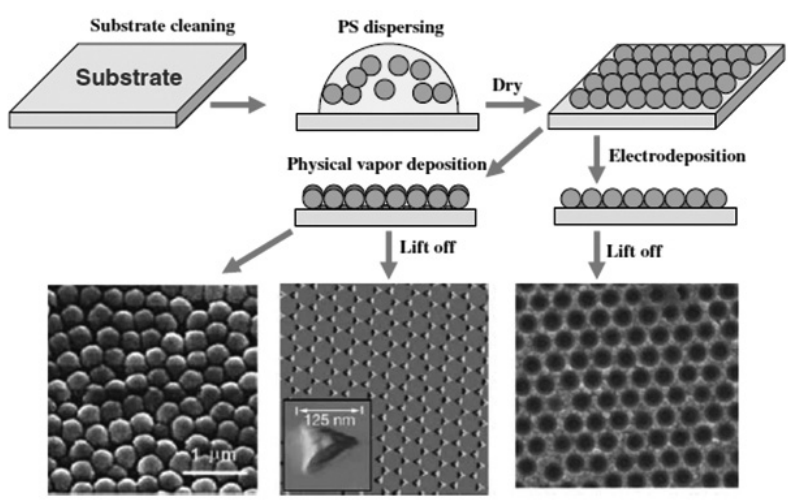

Figure 2: Schematic diagram of template methods using nanosphere lithography to fabricate ordered nanostructured SERS-active substrate Reproduced with permission from: X.-M. Lin, Y. Cui, Y.-H. Xu, B. Ren, Z.-Q. Tian. Analytical and Bioanalytical Chemistry 394, 1729-1745 (2009). 
Since the optical enhancement in SERS is plasmon driven, it can be applied to all molecular systems, i.e. SERS is not molecule specific. This is confirmed in the compilation of molecules displaying SERS signa ${ }^{44}$ and the database available in www.spectroscopynow.com. Notably, the plasmonic enhancement has been confirmed even for molecules with very low scattering cross section such as water and alkanes. However, the challenge of producing reliable, reproducible SERS substrates with an expected enhancement factor remains for the nanostructure fabrication; that would allow one to routinely apply the capabilities of SERS as an analytical too ${ }^{45}$.

Here, we focus the discussion on the SERS/SERRS results obtained for controlled molecular coating of the enhancing nanostructure using the Langmuir-Blodgett (LB) technique ${ }^{46,47}$ for molecules that can form LB films or mixed LB films (LB-SERS). It is, therefore, possible to monitor the surface coverage of the metal using well defined monolayers, allowing to compare SERS substrates and to estimate the enhancement factor for the target molecule. This review paper is organized as follows. The first sections present a discussion of the enhanced SERS and SERRS spectra. Then, the advantages and limitations of the SERS/SERRS work with LB films are given.

\section{SERS/SERRS or plasmon enhanced inelastic scattering}

The molecular vibrational fingerprints for characterization are obtained from two complementary techniques: infrared absorption spectroscopy and Raman (inelastic) scattering. Both the infrared absorption and the inelastic scattering can be enhanced using plasmonic. "An inelastic scattering process produces secondary light quanta with different energy. One such process is the Raman effect. During the interaction of the primary light quantum with a molecule or crystal, the energy of vibrational and/or rotational states may be exchanged and a secondary light quantum of lower or higher energy is emitted. The energy difference is equal to the vibrational energy $E_{v i b}$ of a molecule or crystal and/or the rotational energy $E_{\text {rot }}$ of a molecule. It may be recorded, if monochromatic radiation is used for the primary excitation, as a vibrational, rotational or rotationvibration Raman spectrum." (IUPAC 1997).

The induced dipole produced by the monochromatic electromagnetic radiation (a laser line) used in a Raman experiment is: $p=\alpha E$ [1], where $\mathrm{a}$ is the molecular polarizability (tensorial and vector notations are omitted for the sake of simplification). The molecular polarizability tensor component is a function of the vibrational motions within the molecule (time dependence), and $\alpha$ can be expanded in a Taylor's series near the equilibrium ${ }^{48}$. For each vibrational coordinate $Q_{k}=Q_{k}^{0} \cos \left(\omega_{k} t\right)$ [2], we can write:

$$
\alpha_{k}=\alpha_{0}+\left(\frac{\partial \alpha}{\partial Q_{k}}\right)_{0} \delta Q_{k}+\left(\frac{\partial \alpha^{2}}{\partial Q_{k}^{2}}\right)_{0} \delta Q_{k}^{2}+\ldots
$$

where the first term $\alpha_{0}$ will give rise to the elastic Rayleigh scattering, and the first derivatives in the second term are related to the fundamental Raman vibrational frequencies. The third term corresponds to overtones followed by combinations (not shown). When the expression of polarizability is combined with $p=\alpha E$, the terms for the Rayleigh and the fundamental vibrational frequencies are obtained:

$$
p=\alpha_{0} E_{0} \cos \left(\omega_{0} t\right)+\frac{1}{2}\left(\frac{\partial \alpha}{\partial Q_{k}}\right)_{0} Q_{k}^{0} E_{0}\left[\left(\cos \left(\omega_{0}-\omega_{k}\right) t\right)+\left(\cos \left(\omega_{0}+\omega_{k}\right) t\right)\right]
$$

where $p$ is the oscillating Hertzian dipole. Therefore, the incoming radiation is scattered by the molecule with a slightly different energy and two sets of bands are observed on either side of the elastic scattering band (Rayleigh scattering): Stokes bands of lower energy and anti-Stokes bands of higher energy. When the exciting radiation is in resonance with the electronic absorption of the molecule, i.e. the laser light may also be absorbed by the molecule, then the inelastic scattering is termed resonance Raman scattering (RRS). RRS has a much larger cross section than the spontaneous Raman scattering effect ${ }^{48,49}$.

The SERS signal is due to the enhancement in the near field at the surface of the metal nanoparticles under plasmon excitation, leading to an enhancement factor proportional to $E^{4}{ }^{50}$. The intensification of the electric field on the surface of metal particles on which molecules are adsorbed is treated with the classical theory of electromagnetism by solving the Maxwell equations. Under proper excitation, noble metal nanostructures can exhibit localized collective electronic excitations or LSPR. While these modes present no fundamental differences with the volume plasmons of basic physics textbooks, the different boundary conditions that apply to the Maxwell equations give rise to a geometrically determined behavior. The fields induced at the surface, particularly at excitation in or close to the resonance of these LSPR, are responsible for the enhancement in SERS ${ }^{51,52}$. As already mentioned, this enhancement depends essentially on the dielectric function of the metal at the excitation frequency, the dielectric constant of the medium that involves the surface and the shape, size and packing of the metal particles ${ }^{15,53}$. The control of these parameters is intimately related to the success or failure of producing reproducible SERS substrates.

Experimentally, a necessary condition to achieve SERS is the excitation of LSPR. For laser lines within the visible range of the electromagnetic spectrum, the noble metals $\mathrm{Au}, \mathrm{Ag}$ and $\mathrm{Cu}$ are the most efficient. The plasmon absorption of a $6 \mathrm{~nm} \mathrm{Ag}$ evaporated film is shown in Figure 3, where the most commonly used laser lines at 514.5; 632.8 and $785 \mathrm{~nm}$ are also marked.

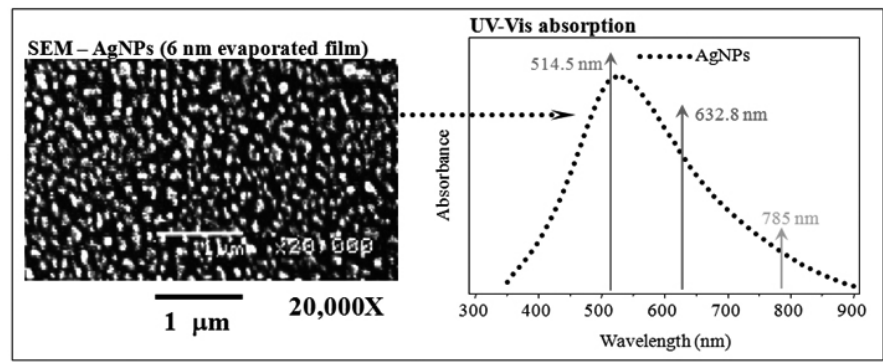

Figure 3: On the left a scanning electron microscopy image of a $6 \mathrm{~nm} \mathrm{Ag}$ evaporated film is shown, while at the right the ultraviolet-visible absorption spectrum is shown. The arrays indicate some common laser lines used in the visible range to achieve the SERS effect.

The interaction among the incoming laser, metal nanoparticles and adsorbed molecule and their relation with spontaneous Raman scattering and SERS are presented as simplified illustrations in Figure 4. Basically, the combination of the electric field from the laser light $\left(\mathrm{E}_{\text {laser }}-\right.$ Figure $\left.4 \mathrm{a}\right)$ and the electric field from the metal nanoparticle $\left(\mathrm{E}_{\mathrm{p}}-\right.$ Figure $\left.4 \mathrm{~b}\right)$ comprises $\mathrm{E}_{\mathrm{Loca}}$ at the surrounding of the metal particles. These two components of $E_{\text {Local }}$ affect directly the magnitude of the induced dipole moment of the molecule $\left(p_{1}\right)$ that is adsorbed at the metal particle surface (Figure 4c). In other words, the molecule is polarized by $\mathrm{E}_{\text {Local }}$ when it is placed on the metal surface and, as a result, the dipole $\left(p_{1}\right)$ is induced. The electric field radiated by this induced molecular dipole $\left(\mathrm{p}_{1}\right)$ can also polarize the metal, inducing a dipole in the metal nanoparticle $\left(\mathrm{p}_{2}\right)$ that oscillates with the same frequency of the induced molecular dipole $\left(\mathrm{p}_{1}\right)$.

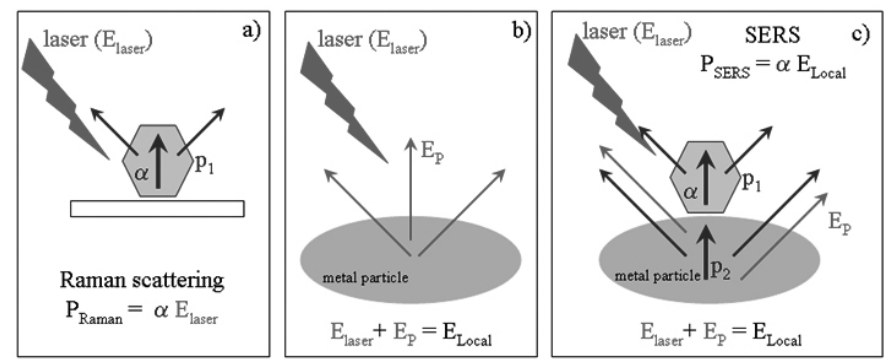

Figure 4: Illustrations showing (a) the dipole moment of the molecule ( $p_{\text {, }}$ ) induced by the incoming laser line in the spontaneous Raman scattering; (b) $\mathrm{E}_{\mathrm{Local}}$ at the surrounding of the metal particles composed by the electric fields from the metal nanoparticle ( $\left.\mathrm{E}_{\mathrm{p}}\right)$ and from the incoming laser $\left(\mathrm{E}_{\mathrm{Lace}}\right)$; (c) SERS effect - the molecule placed at the metal surface is polarized by $\mathrm{E}_{\text {Local }}$ inducing the molecular dipole $\left(p_{1}\right)$ whose radiated electric field can also polarize the metal, inducing a dipole in the metal nanoparticle $\left(\mathrm{p}_{2}\right)$ that oscillates with the same frequency of $\mathrm{p}_{1}$.

A simple model, assuming a spherical metal particle as in Figure 5, is useful to understand this process. For a radius (r) smaller than the wavelength of the incoming radiation (1), the electric field can be considered uniform through the particle and the surface plasma as an oscillating dipole $\left(\mathrm{p}_{\text {metal }}\right)$. So, the Rayleigh electrostatic approximation (magnetic effects are neglected) is satisfied. In 
this case the intensity of $p_{\text {metal }}$ is given by relation [5] where $e_{\text {metal }}$, which is a function of the frequency ( $\mathrm{w}$ ), corresponds to the metal dielectric function, $\mathrm{e}_{\text {medium }}$ is the dielectric constant of the environment that surrounds the metal surface, and $\mathrm{E}_{\text {laser }}$ is the electric field of the incoming radiation that oscillates with a frequency $\mathrm{w}_{\text {laser }}$. The dipole $\mathrm{p}_{\text {metal }}$ is then maximum for a frequency $\mathrm{w}=$ $\mathrm{W}_{\text {laser }}$ in the resonance condition, which means $\operatorname{Re}\left(\mathrm{e}_{\text {metal }}=-2 \mathrm{e}_{\text {medium }}\right)[6]$.

$$
p_{\text {metal }}=\varepsilon_{\text {medium }} \frac{\varepsilon_{\text {metal }}-\varepsilon_{\text {medium }}}{\varepsilon_{\text {metal }}+2 \varepsilon_{\text {medium }}} 4 \pi r^{3} E_{\text {laser }}
$$

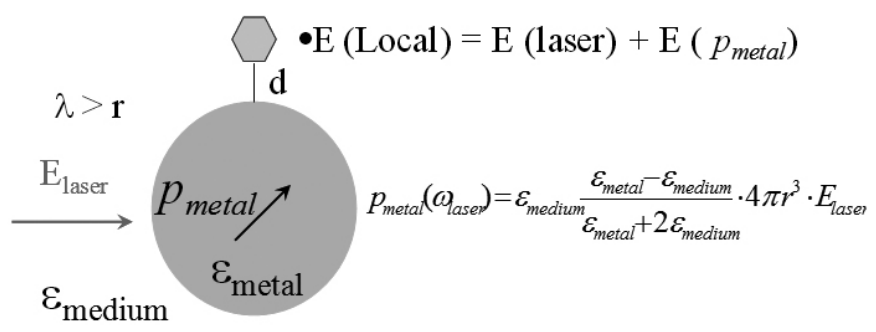

Figure 5: Illustration of the model considering a spherical metal particle, with the local electric field $\left(\mathrm{E}_{\text {Local }}\right)$ established at the environment of the metal particle in the SERS effect.

In addition, the dipole $\mathrm{p}_{2}$ induced in the metal particle oscillating with the same frequency of the induced molecular dipole $p_{1}$ is also maximized because this vibration frequency, which corresponds to the Raman scattering ( $\mathrm{w}_{\text {Raman }}$ ), is close to the laser frequency, i.e. $\mathrm{w}_{\text {laser }} \sim \mathrm{w}_{\mathrm{R} \text { man }}$, in a way that the resonance condition (relation [6]) is also obeyed. This $p_{2}$ is the origin of the main contribution to the Raman signal amplification. In the case of spontaneous Raman scattering only $\mathrm{p}_{1}$ contributes to the signal. In general, not only SERS benefits from this electromagnetic effect but also other processes such as emission, absorption and non-linear effects ${ }^{54,55}$. The SERS effect is highly distance dependent, and is eliminated when the distance $\mathbf{d}$ in Figure 5 reaches around $150 \AA^{56}$.

Although the single nanoparticle is used to give a simple explanation of the EM enhancement mechanism, in practice, SERS/SERRS is observed thanks to the contribution from the highest field or hot spot found in small nanoparticle dimers and aggregates in which interstitial sites are the super-enhancing locations. Modelling of these nanostructures using the electromagnetic theory is computationally demanding and several approaches have been developed that are becoming widely used with varying degree of success. The simplest model for the junction is that of two closely spaced spheres that can be tackled using extended Mie theory ${ }^{57,58}$. The near field enhancement can be estimated and directly related to SERS and surface-enhanced fluorescence (SEF $)^{59}$. The field enhancement on metal nanoparticles in the time domain can be simulated using finite-difference time-domain method (FDTD), or a combination of the FDTD and the pseudo-spectral time-domain method (PSTD) ${ }^{60}$. A very powerful method to simulate light scattering by arbitrarily shaped particles is the discrete dipole approximation (DDA), and a review with applications appears in ref. ${ }^{61}$. Recently, an appealing electrostatic method has been developed, namely the "plasmon hybridization method" $"$, that could be seen as an electromagnetic analog of familiar molecular orbital theory. The latter model describes the plasmon response of complex nanostructures of arbitrary shape. A calculation of the near field distribution for Ag dimer is shown in Figure 6. Here, the highest field (hot spot) is clearly located in the space between the two nanoparticles separated by $2 \mathrm{~nm}$.
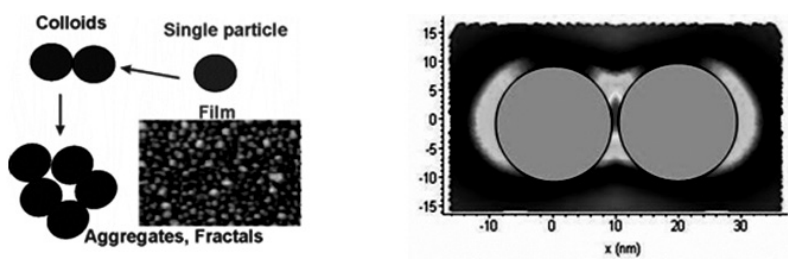

Figure 6: On the right hand side the simulation shows two Ag spheres with an enhanced electric field (in black) between the spheres. On the left figure, representations are given of different states of aggregation for the metal nanoparticles.
3. SERS and SERRS from nanostructures coated with LangmuirBlodgett films

Langmuir films ${ }^{47}$ are formed by spreading a small number of molecules in volatile solutions onto the water subphase in a Langmuir trough. After the evaporation of the solvent, the floating molecules are compressed by barriers forming a monolayer at the air/water interface, i.e. the Langmuir film, as shown in Figure 7a. This process is controlled by measuring the surface pressure vs mean molecular area isotherms ( $\mathrm{p}-\mathrm{A}$ isotherms). Basically, the surface pressure (p) is given by the difference between the surface tension of the water subphase in the absence of the monolayer $\left(\mathrm{g}_{0}\right)$ and the surface tension of the water subphase with the monolayer (g), i.e.:

$$
\pi=\gamma_{0}-\gamma
$$

The most common molecules used to produce Langmuir films are amphiphilic (shown in the inset in Figure 7b), which possess hydrophobic groups responsible for keeping the molecules floating and hydrophilic groups that allow for spreading over the air/water interface. In analogy to P-V isotherms, during compression of the Langmuir monolayers, thermodynamic phases are identified which have been named gas, liquid and solid phases as indicated in Figure $7 \mathrm{~b}$. These phases are directly related to the packing of the molecules at the air/water interface. For instance, in the gas phase the molecules are randomly dispersed while in the condensed phase the molecules are closely packed.

The Langmuir-Blodgett (LB) films $\mathrm{s}^{46,47}$ are produced by transferring the Langmuir monolayers from the air/water interface onto solid substrates as illustrated in Figure 7c. During transfer the surface pressure is kept constant (usually within the condensed phase of the Langmuir film) and the substrate is immersed and withdrawn by means of a dipper into the water subphase. When the Langmuir film is transferred only during the upstrokes, the LB films are referred to as Z-type. The films transferred only in the downstrokes are the $\mathrm{X}$-type films, and for transfer in both immersion and withdrawal Y-type LB films are formed. It should be stressed that during transfer the barriers are moved to keep the surface pressure constant. The ratio between the decrease in area on the Langmuir trough within the barriers and the area of the substrate covered by the LB film is the transfer ratio (TR), which is essential for controlling the LB film deposition. Ideally, TR should be 1 , for which the hydrophilicity of the substrate and the speed of the dipper play important roles.

\section{(a) Langmuir film}

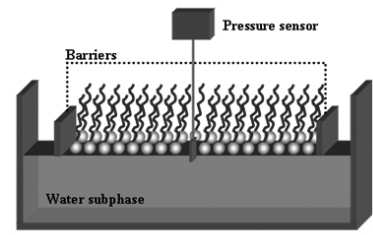

(b) $\pi$-A isoterms

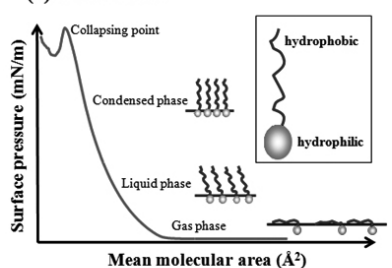

(c) LB films

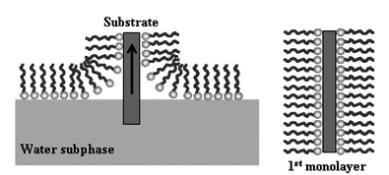

Figure 7: (a) Langmuir film (condensed phase) formed at the air/water interface using a Langmuir trough and amphiphilic molecules; (b) representation of a characteristic $\pi-\mathrm{A}$ isotherm for a Langmuir film of amphiphilic molecules (inset) presenting the gas, liquid and condensed phases, and the collapsing point; (c) transferring of the Langmuir monolayer from the air/water interface onto solid substrates forming the LB film. 
The Raman scattering technique has been used with LB films since the first half of the 1970's. ${ }^{63}$ In 1985 Aroca et al.$^{64}$ reported the SERS effect of a metalfree tetra-tert-butylphthalocyanine $\left((\mathrm{t}-\mathrm{bu})_{4} \mathrm{H}_{2} \mathrm{Pc}\right) \mathrm{LB}$ monolayer deposited onto evaporated In and $\mathrm{Ag}$ island films, while Chen et al. ${ }^{65}$ presented SERS results for LB monolayers and bilayers of polydiacetylene deposited onto evaporated $\mathrm{Ag}$ island films. The SERS signal was $10^{2}$ times the RRS signal for both In and $\mathrm{Ag}$ islands [56], consistent with enhancement factors for other molecules. Chen et al. ${ }^{66}$ also applied RRS and SERS to study structural and electronic properties of polydiacetylene forming LB monolayer and multilayers. These results represent the first observation of a "disorder to order" transformation in an LB film, which was assigned to the interactions between the first and the subsequent layers.

The possibility of working at controlled diluted levels (monomolecular scale) and well-defined molecular spatial distribution provided by the LB technique was important for the development of theoretical and experimental studies to elucidate the SERS effect. The distance dependence of the moleculemetallic substrate for SERS was studied by Kovacs et al. ${ }^{56}$ with LB films of arachidic acid as spacer layers to control the distance between evaporated In and Ag island films and LB monolayers of a highly substituted phthalocyanine. This approach was possible due to the large SERS cross section of phthalocyanine and the low SERS cross section of arachidic acid. A fairly quantitative agreement was found between the calculation and experimental results for both the distance and magnitude of the enhancement, as shown in Figure 8. Experiments carried out with three exciting laser lines show that a distance of ca. $150 \AA$ is sufficient to extinguish the enhancement. Similar results were reported by Cotton et al. ${ }^{67}$.
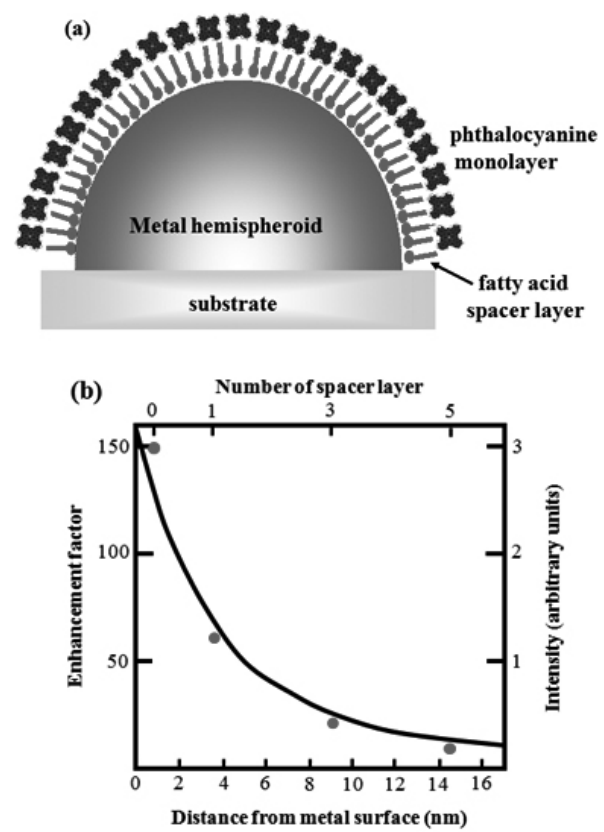

Figure 8: (a) Schematic view of a hemispheroid metal particle covered by an LB monolayer of arachidic acid (spacer layer) and an LB monolayer of phthalocyanine; (b) enhancement factor calculated for In islands using the electromagnetic model (solid line) as a function of distance from the spheroid surface. The experimental points are based on the enhancement factors for the intensity of the $686 \mathrm{~cm}^{-1}$ line of the phthalocyanine LB monolayer. The wavelength of the exciting laser light was $618 \mathrm{~nm}$. Adapted from reference ${ }^{56}$.

Using LB films also made it possible to study how the SERS effect depends on the surface coverage of the metal by the adsorbed dye. Aroca et $a l .{ }^{68}$ produced mixed LB monolayers of phthalocyanine and arachidic acid onto Ag-coated $\mathrm{Sn}$ spheres, evaporated Ag and Au island films to enhance the Raman signal. Notably, the maximum enhancement was achieved when the surface coverage was below a monolayer of the dye. Kim et al. ${ }^{69}$ also examined the relationship between surface coverage and SERRS for a cyanine dye (S-120) dispersed in LB monolayers containing arachidic acid and methyl arachidate on evaporated $\mathrm{Ag}$ island films. The results obtained with this film architecture illustrated in Figure 9a indicated that the SERRS intensity was maximized at ca. $8 \%$ of the dye, as shown in Figure $9 \mathrm{~b}$. This dependence on the coverage might be related to changes in the medium dielectric function and/or dipole-dipole dye interaction.

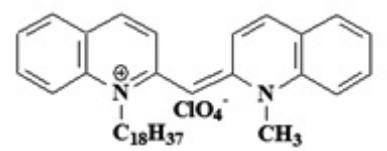

(a)

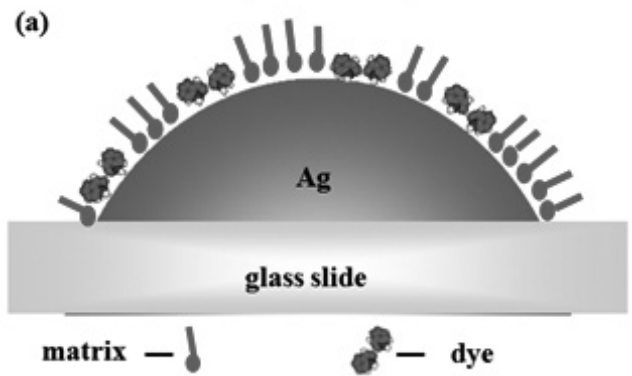

Figure 9a: Molecular structure of S-120 and a schematic diagram of the dye-lipid monolayer on the Ag-coated glass slide. The dye and lipid representations are shown approximately to the same scale as the Ag particle (ca. $400 \AA$ diameter). Adapted from reference ${ }^{69}$.
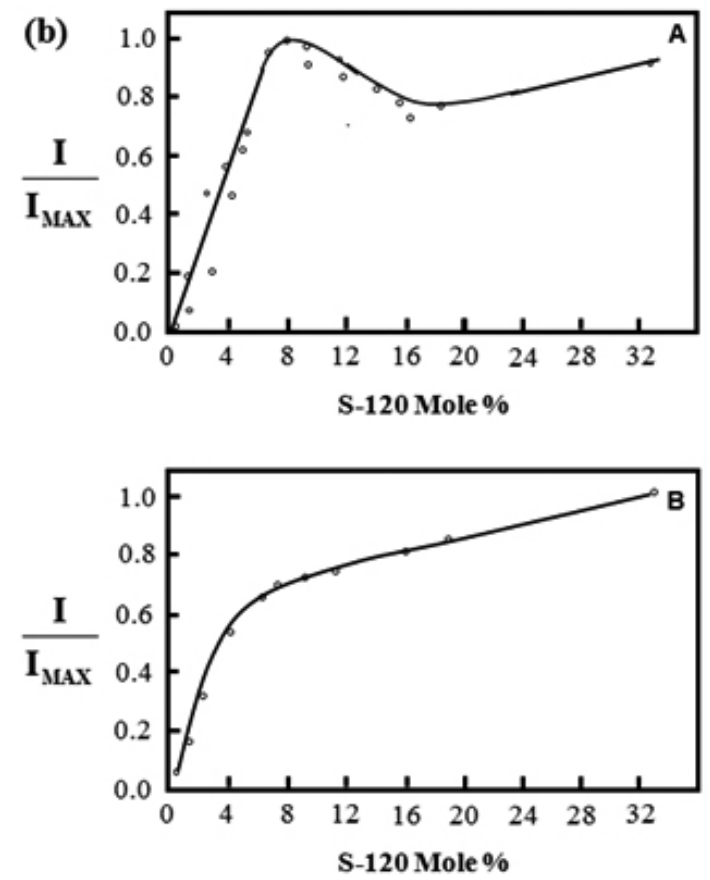

Figure 9b: Intensity of the SERRS band at $1360 \mathrm{~cm}^{-1} v s$ mole $\%$ of dye in the lipid matrix. Intensities have been normalized to the maximum value. Part $\mathrm{A}$ is for data acquired with one scan. The solid and dotted lines correspond to two different batches of evaporated Ag island films. Part B is for data acquired by adding 30 scans. Adapted from reference ${ }^{69}$.

The previous examples illustrate how the LB technique has been instrumental to understand SERS mechanisms. At the same time SERS and SERRS have been applied to study a variety of LB film properties. For instance, they were used to determine the molecular organization and/or molecular aggregation ${ }^{70-76}$, to distinguish dye monomer and aggregates ${ }^{77}$, in molecular recognition $^{78,79}$ and interactions between LB film and distinct analytes either in gas or solution ${ }^{42,71,76,80,81}$. The reversible adsorption of $\mathrm{NO}$ on phthalocyanine LB monolayers deposited on $4 \mathrm{~nm}$ evaporated Au island films was monitored with SERRS spectroscopy ${ }^{82}$. In a study with three phthalocyanines, namely $\mathrm{H}_{2}$ TTPc (metal-free tetratert-butylphthalocyanine), CuTTPc (copper tetra-tert- 
butylphthalocyanine) and $\mathrm{YbPc}_{2}$ (ytterbium bisphthalocyanine), it was shown that the interaction with the electron-accepting gas affects the intensity of the vibrations of the pyrrole moieties and depends on the central metal atom. The NO-phthalocyanine interaction increases according to the sequence $\mathrm{H}_{2} \mathrm{TTPc}<$ CuTTPc $<\mathrm{YbPc}_{2}$. Figure 10 shows a pronounced change induced by $\mathrm{NO}_{\mathrm{x}}$ in the relative intensities of the stretching frequencies of the macrocycle in the SERRS spectra of $\mathrm{YbPc}_{2}$

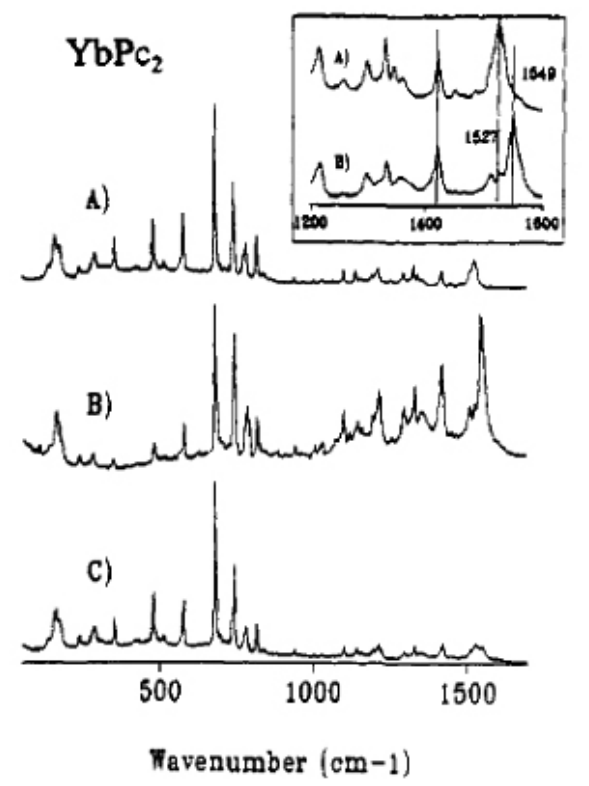

Figure 10: SERRS spectra of an LB monolayer of $\mathrm{YbPc}_{2}$ on $\mathrm{Au}(\mathrm{A})$, after adsorption of $\mathrm{NO}_{\mathrm{x}}(\mathrm{B})$ and recovery after gas desorption $(\mathrm{C})$. The pyrrole frequency region is expanded in the inset. Reproduced with permission from: C. Li, J. Huang, Y. Liang. Langmuir 16, 7701-7707 (2000).

Molecular-level interactions between phospholipids and methylene blue (MB) in LB films have also been investigated with SERRS ${ }^{83}$. In order to deposit multilayer LB films of dipalmitoyl phosphatidyl glycerol (DPPG), an aqueous subphase containing low concentrations of the cationic polyelectrolyte poly(allylamine hydrochloride) (PAH) was used. This strategy allows characterization of mixed DPPG+PAH LB films through cyclic voltammetry and impedance spectroscopy in the presence of MB solution. The nature of the interaction between DPPG and MB was inferred by $\pi$-A isotherms, whose data in Figure 11a reveals a stronger affinity between DPPG and MB than between DPPG and PAH. The latter was sufficient to transfer MB trapped within the DPPG layers in the LB films; a schematic diagram of the structuring of the monolayer is given in Figure 11b. Despite the possibility of growing multilayer LB films, a mixed DPPG+PAH+MB monolayer transferred onto evaporated $\mathrm{Ag}$ island films was already enough to determine the electrostatic nature of the interaction between DPPG and MB via SERRS. Figure 11c shows a SERRS line mapping for the mixed DPPG $+\mathrm{PAH}+\mathrm{MB}$ LB monolayer by combining the Raman spectrograph and optical microscopy. In this case 101 spectra were collected point-by-point (step of $1 \mu \mathrm{m}$ ) for a line of $100 \mu \mathrm{m}$, which is shown in the optical image in Figure 11d. The inset in Figure 11d brings a SEM image for the Ag nanostructures used as SERS-substrates. This possibility of recording Raman images has been proven powerful to study the LB film interface or morphological features ${ }^{32,73-75,83,84}$.
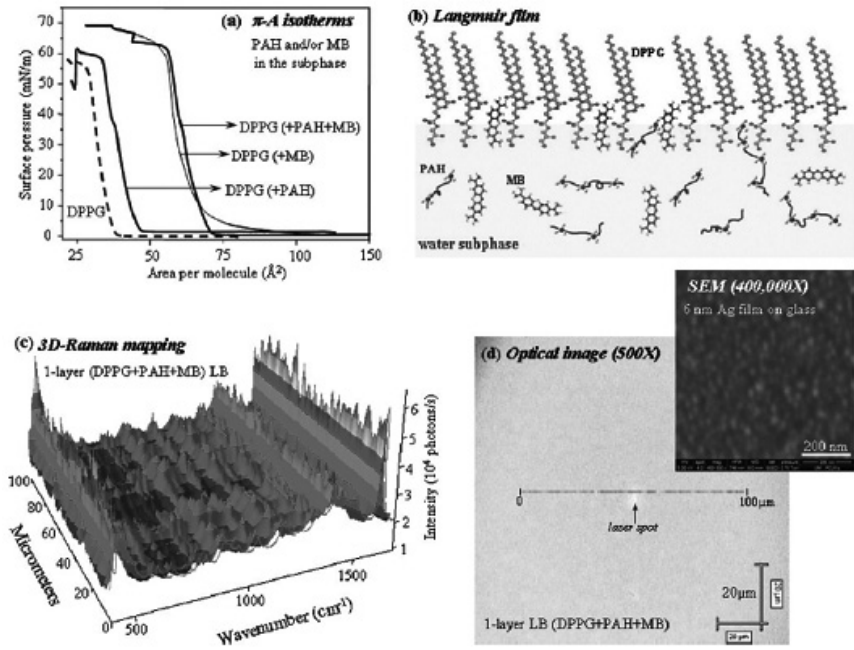

Figure 11: (a) DPPG $\pi$-A isotherms recorded for the water subphase containing either PAH $(0.01 \mathrm{mg} / \mathrm{mL})$ or MB $\left(64 \times 10^{-6} \mathrm{mg} / \mathrm{mL}\right)$. (b) Scheme illustrating the DPPG Langmuir film at the air-water interface with PAH and MB dissolved in the subphase. (c) SERRS spectra recorded for the onelayer LB film for the DPPG transferred onto a $6 \mathrm{~nm}$ Ag film with the water subphase containing $0.01 \mathrm{mg} / \mathrm{mL}$ PAH and $64 \times 10^{-6} \mathrm{mg} / \mathrm{mL}$ MB. (d) Optical image of this one-layer LB film on a $6 \mathrm{~nm} \mathrm{Ag}$ film with the line from where the SERRS spectra were collected point-by-point. The inset is a scanning electron microscopy image showing the Ag nanoparticles formed by evaporation of 6 $\mathrm{nm} \mathrm{Ag}$ onto a glass substrate. In all cases, the SERRS spectra are assigned to MB. Reproduced with permission from: P. H. B. Aoki, P. Alessio, M. L. Rodriguez-Mendez, J. A. D. Saez, C. J. L. Constantino. Langmuir 25, 1306213070 (2009)

Aroca and Constantino ${ }^{32}$ were the first to report SERS and SERRS images (mapping and global image) for an LB monolayer. A perylene derivative mixed to arachidic acid ( $20 \%$ and $1 \%$ molecular concentration of perylene) was deposited onto an evaporated $\mathrm{Ag}$ island film. Figure 12 shows at the top SERRS area mappings for the neat and $20 \%$ mixed LB monolayers. These area mappings were built collecting SERRS spectra point-by-point from spots of ca. $1 \mu \mathrm{m}$ in diameter for an area of $40 \times 40 \mu \mathrm{m}$. The brighter spots represent higher intensities for the SERRS band at $1296 \mathrm{~cm}^{-1}$, which is characteristic of the perylene derivative. Therefore, the latter reveals that the perylene is more homogeneously dispersed when forming monolayers in a matrix of fatty acid than in neat monolayers. A SEM image of the Ag island film with $6 \mathrm{~nm}$ mass thickness (SERS-susbtrate) is shown at the bottom (left corner). A line mapping built by collecting SERRS spectra every $1 \mu \mathrm{m}$ along $100 \mu \mathrm{m}$ is shown at the bottom right corner for the $1 \%$ mixed LB monolayer. The latter result is important because at this \% the signal is generated by less than 40,000 perylene molecules and, because the signal-to-noise ratio is ca. 100 , it could be concluded that the detection limit for this perylene derivative was in the class of molecules for which SMD could be achieved. This work opened the avenue for SMD by Aroca's group as will be seen in the next section. 
$20 \%$ Mixed
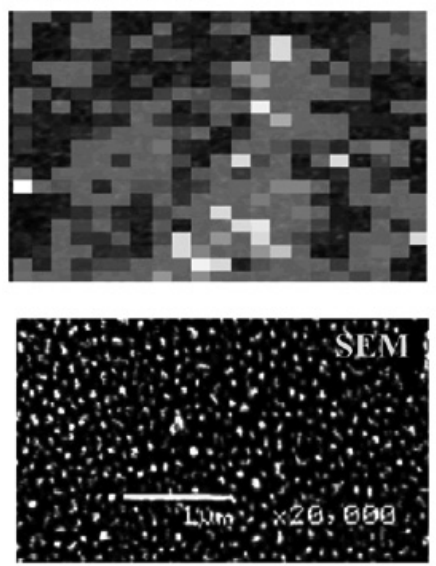
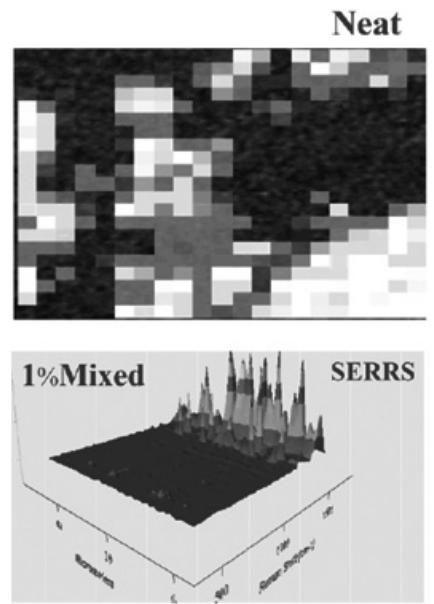

Figure 12: top - area maps ( $40 \mu \mathrm{m} \times 40 \mu \mathrm{m})$ showing the point-by-point variation in the SERRS (514.5 $\mathrm{nm}$ irradiation laser line) signal for the baselinecorrected vibrational peaks at $1296 \mathrm{~cm}^{-1}$. The map at the top left corresponds to the $20 \%$ mixed perylene derivative-arachidic acid LB monolayer and at the top right to the neat perylene derivative LB monolayer (both on evaporated $\mathrm{Ag}$ island films). Bottom - SEM image (20,000X) of the evaporated Ag island film (left) and the SERRS line mapping for $1 \%$ mixed LB monolayer (right). Adapted from reference ${ }^{32}$.

Other applications of SERS involve basic spectroscopic studies to infer chemical information through vibrational assignments ${ }^{81,85-90}$. Berno et al. ${ }^{91}$ studied the effect of the laser line excitation energies $(1064 ; 647.1 ; 514.5$ and $488 \mathrm{~nm}$ ) on enhanced Raman spectra (in and out of resonance with the target molecule), some of these spectra are shown in Figure 13. One and two LB layers of $\mathrm{EuPc}_{2}$ (europium bisphthalocyanine) were deposited onto evaporated substrates containing $\mathrm{Ag}(6 \mathrm{~nm})$ for SERS experiments and Au (20 nm) for FTSERS experiments. The latter was presented for the first time.

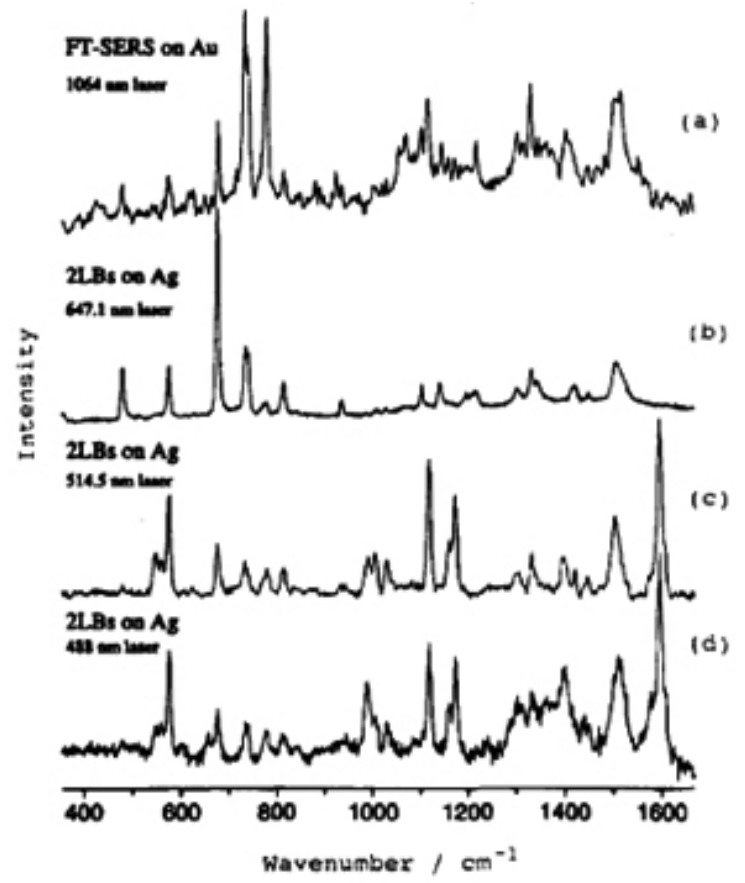

Figure 13: SERS spectra of EuPc ${ }_{2}$ - (a) one LB monolayer on $20 \mathrm{~nm} \mathrm{Au}$ island film; (b)-(d) two LB monolayers on $6 \mathrm{~nm} \mathrm{Ag}$ island film. Reproduced with permission from: B. Berno, A. Nazri, R. Aroca. Journal of Raman Spectroscopy 27, 41-47 (1996).
Studies involving optical properties at different temperatures have also been reported. Johnson et al. ${ }^{92}$ (see comment below Figure 14) applied SERRS spectroscopy to LB monolayers of PDCI (N-octyl-3,4-perylenedicarboximide) deposited onto $6 \mathrm{~nm}$ evaporated Ag island films. SERRS was recorded at 95 and $293 \mathrm{~K}$ as shown in Figure 14, from which it could be concluded that there was a long-range molecular organization in the LB films and that the degree of alignment increased with decreasing temperatures.

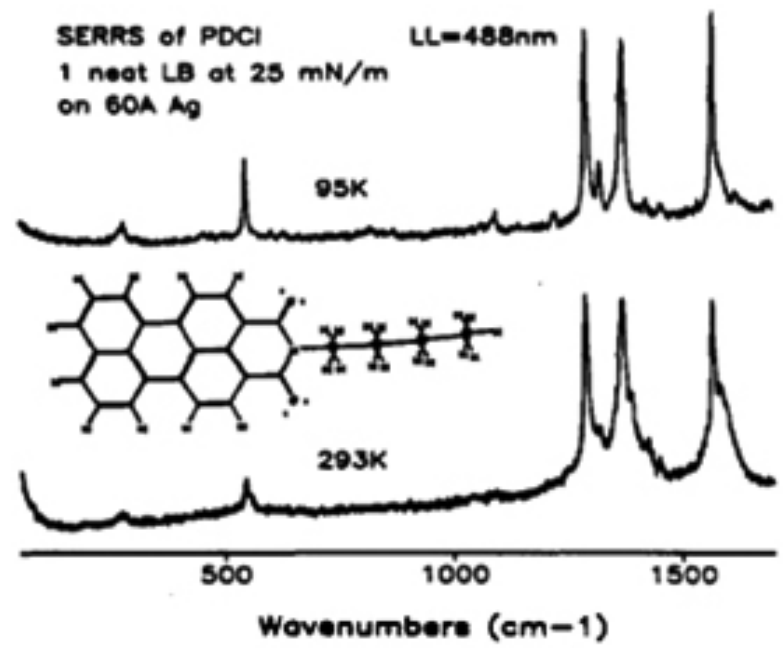

Figure 14: SERRS spectra of an LB monolayer of neat PDCl transferred at $25 \mathrm{mN} / \mathrm{m}$ on evaporated $\mathrm{Ag}$ island film recorded at different temperatures. Reproduced with permission from: E. Johnson, R. Aroca, Y. Nagao. Journal of Physical Chemistry 95, 8840-8843 (1991).

The LB technique has also been used as a strategy to produce SERSactive substrates ${ }^{93-96}$. Mahmoud et al. ${ }^{95}$ developed a platform to study SERS from adsorbed molecules on nanoparticle surfaces. For instance, $50 \mathrm{~nm} \mathrm{Ag}$ nanocubes were deposited onto quartz substrate at different surface pressures, thus yielding LB monolayers with distinct interparticle distances shown in Figure 15. The natural aggregation of the nanocubes in the LB monolayer is beneficial for SERS spectroscopy as depicted in Figure 16, since aggregation leads to a broad localized surface plasmon band that strongly enhances the scattering of Raman photons.
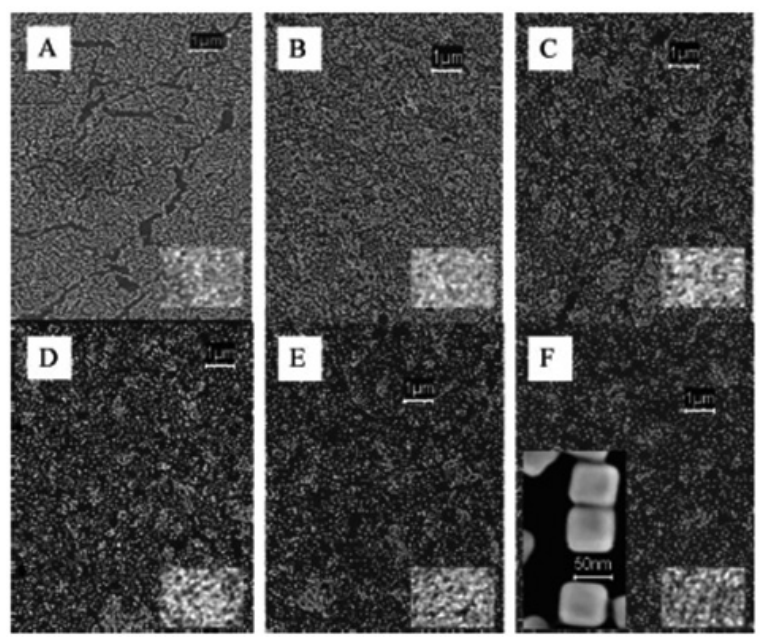

Figure 15: SEM images of Ag nanocubes adsorbed onto quartz substrates prepared at different surface pressures $-25,20,15,10,5$, and $0 \mathrm{mN} / \mathrm{m}(\mathrm{A}-\mathrm{F}$, respectively). The dark field images corresponding to each SEM image are shown in the inset. A high-resolution image of the nanocubes is shown in the inset of (F). Reproduced with permission from: M. A. Mahmoud, C. E. Tabor, M. A. El-Sayed. Journal of Physical Chemistry C 113 (14), 5493-5501 (2009). 


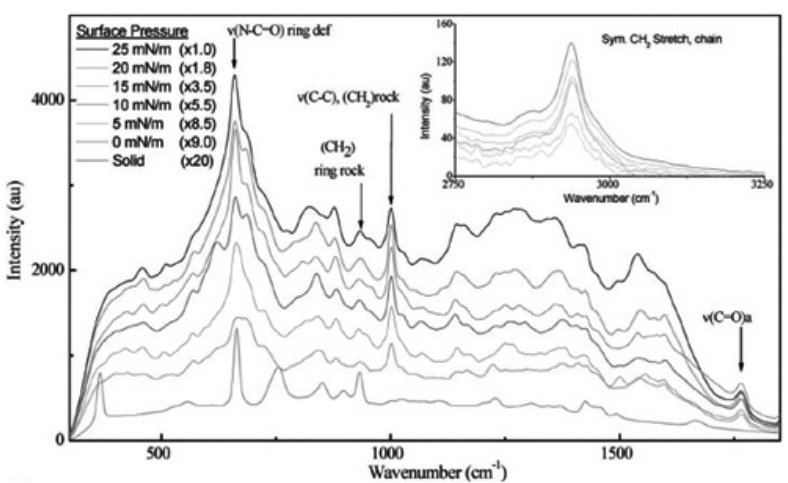

Figure 16: SERS spectra of bulk solid PVP and PVP adsorbed onto the surface of $\mathrm{Ag}$ nanocubes deposited onto a quartz substrate at surface pressures of $0,5,10,15,20$, and $25 \mathrm{mN} / \mathrm{m}$. Adapted from reference ${ }^{95}$. $\mathrm{PVP}=$ poly(vinylpyrolidone).

With regard to the type of molecules used for SERS, pyridine dyes were the first studied. However, there is a wide scope of molecules that have been incorporated into LB films for SERS/SERRS studies. They include mono and bis phthalocyanines ${ }^{56,64,71,80,85}$, polydiacetylene ${ }^{65}$, cyanides ${ }^{69}$, perylenes ${ }^{32}$, $70,77,97-100$, lipids ${ }^{78,90,101,102}, \mathrm{C}_{60}{ }^{103,104}$, drugs such as pirarubicin, adriamycin, daunorubicin and idarubicin, adenosine, thymidine and uridine, methylene blue $^{83,105,106}$, crown ethers ${ }^{93}$, azobenzenes and azopolymers ${ }^{89,94,107-109}$, pyridine and derivatives ${ }^{110}$, rhodamine and derivatives ${ }^{25,111}$, poly-p-phenylenevinylene derivative $\mathrm{e}^{88}$, poly(vinyl) pyrrolidone $\mathrm{e}^{95}$, xanthene dye $\mathrm{e}^{112}$. A detailed discussion of molecule specificity for SERS and a list of molecules studied using SERS or SERRS are found in ${ }^{10}$.

\section{SERS/SERRS and single molecule detection (SMD)}

Single Molecule Spectroscopy (SMS) ${ }^{113}$ represents spectroscopic detection at the ultimate sensitivity level of ca. $1.66 \times 10^{-24}$ moles of the molecule of interest (1.66 yoctomole), a quantity of moles equal to the inverse of Avogadro's number. To achieve a situation in which only one molecule is in resonance, one has to dilute the target molecule to work with roughly $10^{-}$ ${ }^{10} \mathrm{~mole} / \mathrm{liter}$ concentrations, in a probed volume of $10 \mu \mathrm{m}^{3}$. In addition, very important for the work described here is the fact that detection of the single molecule must be done in the presence of million or billions of solvent or host molecules. Also important is the occurrence of noise from the measurement itself.

SMS encompasses several techniques. However, given the large molecular cross section of fluorescence species, fluorescence has been the paramount analytical technique ${ }^{114}$ in SMD experiments, and has been a reference point for the development of SMD via SERS/SERRS. The advantage of the SERS/ SERRS approach is in the rich structural information contained in the Raman scattering itself, enabling SMD with molecular recognition and structural selectivity ${ }^{19,115}$. In one of the seminal papers of SMD-SERS (Kneipp ${ }^{21}$ ), the experiments were carried out using near-infrared excitation at $830 \mathrm{~nm}$ and collecting SERS spectra of crystal violet directly adsorbed onto Ag colloids. It means that the laser line was out of resonance with the electronic absorption of the target molecule. In the second paper (Emory \& $\mathrm{Nie}^{22}$ ) single molecules were detected at room temperature also on $\mathrm{Ag}$ colloids. The dye was rhodamine $6 \mathrm{G}$, and the laser line $514.5 \mathrm{~nm}$, i.e., the effect was enhanced resonance Raman or SERRS.

We have adopted a different approach for SMD, which combines SERS/ SERRS and the LB technique for thin film fabrication ${ }^{20}$. The advantages of the technique have been widely recognized: "In our opinion, the most convincing method based on ultra-low concentrations is possibly the one based on Langmuir-Blodgett monolavers, developed by Aroca and co-workers." (Le $\mathrm{Ru}$, Echegoing \& Meyer. arXiv:physics/0608139 v1 14 Aug 2006). In the case of SERRS, SMD can be achieved by exploiting the multiplicative effect of RRS amplification (up to 6 orders of magnitude) together with the plasmon amplification (up to 7 orders of magnitude in average), leading to (RRS $x$ SERS). In addition, the approach takes advantage of the micrometric spatial resolution in Raman-microscopy, including new spatial mapping capabilities, and high sensitivity available in dispersive systems equipped with CCD detectors. Mapping of the enhancing surface, coated with an LB monolayer, permits to create chemical images that provide a powerful visualization of the variation of the enhancement factor on the two-dimensional nanostructured surface. When the statistics breaks down, a clear image can be created of the detection of the rare event involving the coupling between the molecule and the hot spot. The common effect of photobleaching ${ }^{116}$ has been minimized by working with very low energy density at the sample and short accumulation times. Furthermore, advantage is taken of the precise molecular control provided by the LB technique to produce monolayers containing a certain amount of target molecule per unit area of the LB film deposited onto Au or Ag nanoparticles (usually glass covered by AgNPs or AuNPs, formed by vaccum evaporation). Figure 17 shows a scheme where the single molecule to be detected (target molecule) is in a matrix of fatty acid with an average distribution of 1 molecule per micrometer square. This distribution is based on the spatial resolution given by the objective and the laser line used in microRaman. In summary, using the LB technique, an analyte of interest can be doped into a matrix of spectral silent material (low Raman cross section), allowing a high degree of control of an analyte's concentration per unit of surface enhancing area. Furthermore, by spatially "fixing" the analyte onto the SERS substrate, the dynamics that complicate much of SM-SERS in solution can be avoided. The collected dataset (spectral maps) can then be analyzed to achieve a clearer picture of analyte/substrate interactions uncomplicated by the effect of dynamics.

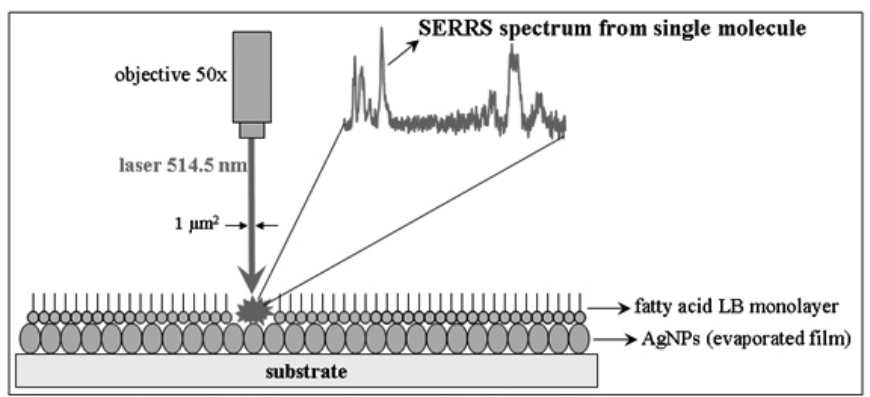

Figure 17: experimental setup applied by Aroca's group to collect SERRS spectra from a single molecule.

The results leading to SMD using SERRS and LB films ${ }^{20,100,117,118}$ are shown in Figure 18, which presents SERRS global images for single molecules of a perylene derivative dispersed in a LB monolayer of arachidic acid. In the global image the laser is defocused reaching an area with diameter of ca. 40 $\mathrm{mm}$ and only the light from a specific wavenumber is collected $\left(1370 \mathrm{~cm}^{-1}\right.$, characteristic of the perylene derivative in this case). Therefore, when the target molecule is absent, only a scattered background is observed instead of bright spots. In 2007, SERS (instead of SERRS) was applied to SMD for the first time using LB films ${ }^{118}$. A step toward SMD in a biological system was taken recently with a labeled phospholipid in a fatty acid LB monolayer on Ag evaporated island films ${ }^{112}$, as shown in Figure 19. One should note that SMD could also be achieved with SERRS in perylene dye films fabricated with the conventional casting method that can be found in a recent review of SMD using SERRS and SERS $^{27}$.

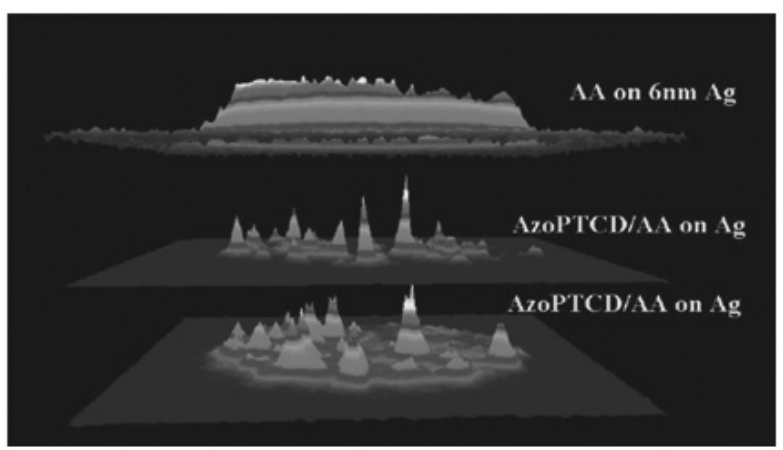

Figure 18: one global image for neat arachidic acid (AA) LB monolayer on $\mathrm{Ag}$ evaporated island film (top) and two global images for perylene derivative single molecule LB monolayer (AzoPTCD/AA), all recorded at $1370 \mathrm{~cm}^{-1}$. Reproduced with permission from: C. J. L. Constantino, T. Lemma, P. A. Antunes, R. Aroca. Analytical Chemistry 73, 3674-3678 (2001). 
(a)

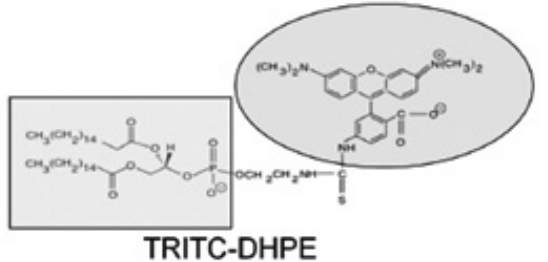

(b)

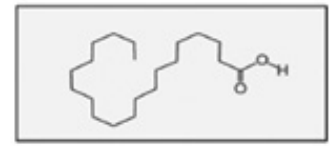

Arachidic Acid

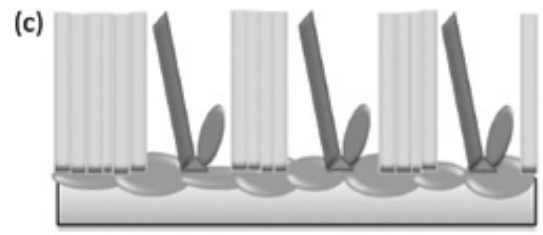

Figure 19a: components of an LB-SERRS film - (a) TRITC-DHPE tagged phospholipid; (b) arachidic acid (AA) as matrix material; (c) illustration of the AA/TRITC-DHPE mixed monolayer on an Ag evaporated film. Reproduced with permission from: N. P. W. Pieczonka, G. Moula, R. F. Aroca. Langmuir 25, 11261-11264 (2009). TRITC-DHPE = N-(6-tetramethylrhodaminethiocarb amoyl)-1,2-dihexadecanoyl-sn-glycero-3-phosphoethanolamine.

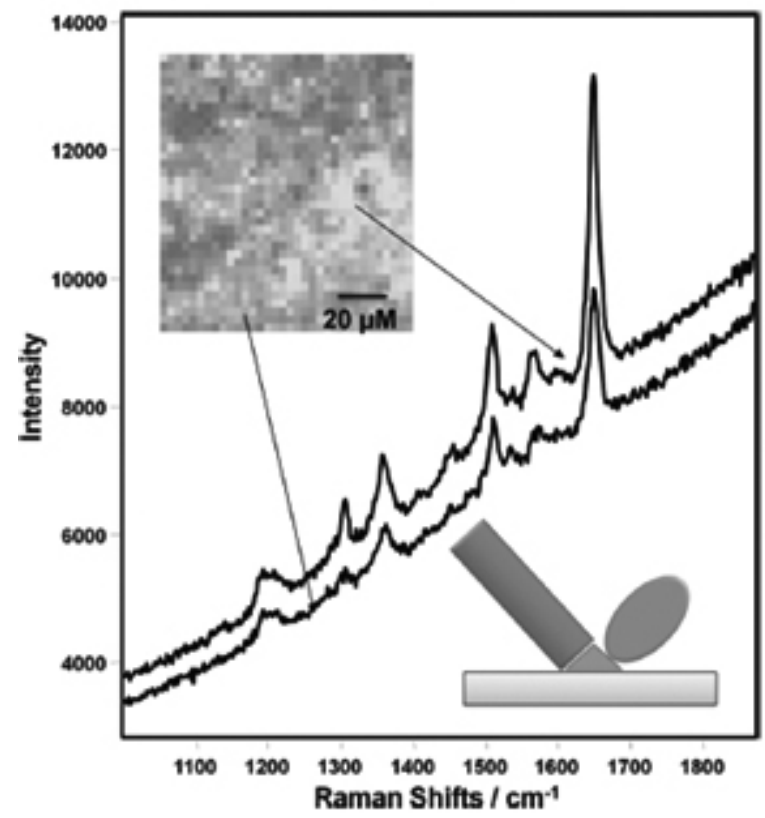

Figure 19b: ensemble SERRS map of a film fabricated with 10:1 AA/ TRITC-DHPE excited at $514.5 \mathrm{~nm}$ and ca. $20 \mu \mathrm{W}$. Step sizes were $3 \mu \mathrm{m} \times 3$ $\mu \mathrm{m}$, and the map was constructed from 1,156 collected spectra. An intensity map was generated from the integrated area of the $1650 \mathrm{~cm}^{-1}$ band. The inset shows the likely orientation of TRITC-DHPE with respect to the metal surface. Reproduced with permission from: N. P. W. Pieczonka, G. Moula, R. F. Aroca. Langmuir 25, 11261-11264 (2009).

The attempts by various groups to reach SMD using SERS led to developments that deserve to be highlighted. In 1988, the SERRS spectra of rhodamine $6 \mathrm{G}(\mathrm{R} 6 \mathrm{G})$ were collected at a concentration of $7 \times 10^{-14} \mathrm{~mol} / \mathrm{L}$ in an $\mathrm{Ag}$ colloid solution containing $\mathrm{NaCl}$, which was added to induce $\mathrm{AgNPs}$ aggregation and therefore extend the absorption band to the near IR. The latter led to ca. 100 R6G molecules within the volume probed by the laser (10
$\mathrm{nL})^{119}$. These results were confirmed in 1995 when ca. 60 R6G molecules were detected within the laser probed volume via SERRS (514.5 nm laser line) for a concentration of $8 \times 10^{-16} \mathrm{~mol} / \mathrm{L}$ of R6G in Ag colloid aqueous solution activated via $\mathrm{NaCl}^{120}$. Still in 1988 , SERS signals were obtained from around 100 pyridine molecules on an $\mathrm{Ag}$ electrode ${ }^{121}$. Taylor et al. ${ }^{122}$ were able to detect crystal violet in Ag colloids using SERRS at concentrations below $10^{-12} \mathrm{~mol} / \mathrm{L}$ whose detection limit was estimated at ca. 600 molecules ( $1 \mathrm{~nL}$ probed volume). In 1996 Rodger et al. ${ }^{123}$ detected ca. 200 rhodamine dye molecules using SERRS from $\mathrm{Ag}$ colloid solutions at $6.87 \times 10^{-18} \mathrm{~mol} / \mathrm{L}$. In $1997 \mathrm{Kneipp}$ et al. ${ }^{21}$ and Nie and Emory ${ }^{22}$ announced the SMD using SERS, which motivated further studies. In 1998 Zeisel et al. ${ }^{124}$ obtained the SERS spectra from approximately 300 dye molecules (cresyl fast violet and R6G) on Ag substrates. In 1999 Xu et al. ${ }^{125}$ detected single hemoglobin $(\mathrm{Hb})$ protein molecules using SERS for a $\mathrm{Hb}$ molecule attached between two AgNPs. Since 2000 Aroca' group has worked on LB films, with detection of $40,000^{32}$ and $400^{126}$ target molecules (distinct dye perylene derivatives), and in 2001 the first article regarding SMD using SERRS and LB films was published ${ }^{20}$. In 2005 Koo et al. ${ }^{127}$ reported SMD for deoxyguanosine monophosphate and deoxyadenosine monophosphate using SERS with coherent anti-Stokes Raman scattering and Delfino et al. ${ }^{128}$ for cytochrome $\mathrm{C}$ in $\mathrm{Ag}$ colloid solution or immobilized onto glass slides using a quasi resonant laser line. In 2006 Kalkan and Fonash reported SMD of fluorescein using SERS and Ag surfaces activated by laser ${ }^{129}$. Zhang et al. ${ }^{130}$ announced in 2009 SMD using SERS for azo dyes (related to human carcinogen) adsorbed on $\mathrm{Ag}$ particle aggregates in colloid, while Blackie et $a l .{ }^{131}$ applied the bianalyte approach to detect adenine through SERS.

The theoretical framework for the phenomenology and statistics of SERS signals from single molecules in the presence of the electromagnetic hot spots was put forward by Le Ru et al. ${ }^{132}$, which could account for various experimental manifestations of the effect reported in the literature.

\section{Final remarks and perspectives}

After the initial reports of the spectrum of a single molecule ${ }^{21,133}$, there has been a concerted effort to demonstrate and establish SERS/SERRS as an analytical technique for single molecule detection (SMD). Single-moleculeSERS and single-molecule-SERRS, carrying the molecular fingerprints in the Raman vibrational information are on the rise, as complementary methods to fluorescence and surface-enhanced fluorescence in the emergent field of single molecule spectroscopy ${ }^{134}$. The use of the LB technique has played an important role in the observation of SERS/SERRS spectra of a single molecule and has contributed to validate single molecule detection in several experiments: i) using doped LB monolayers with an average of one molecule in the field of view of the Raman-microscope ${ }^{135,136}$; ii) LB used in the bi-analyte technique, where two analyte molecules are simultaneously used to confirm the single molecule origin of the observed SERS/SERRS ${ }^{136,137}$. SMD has also been confirmed using isotopes offering unique vibrational signatures for the same electronic state ${ }^{138}$, ${ }^{139}$. The validation of SMD-SERS (SMD-SERRS) led to a consensus on the idea of "hot spots": a spatial location in Ag or Au nanostructure aggregates where the enhancement factor is greater than $10^{7} \cdot{ }^{34}$ Hot spots are described as localized surface plasmon resonances (LSPR), i.e., spatially localized resonances producing nanoscale spatial locations of highly enhanced electromagnetic fields. Hot spots are found in the junctions of aggregated nanostructures such of $\mathrm{Ag}$ and $\mathrm{Au}$; colloidal aggregates or evaporated $\mathrm{Ag}$ island films among others. The potential and advantages of the $2 \mathrm{D}$ LB structures to explore the spectral properties in the transition from the average SERS/SERRS spectra to the single molecule regime have been demonstrated with a large amount of data collected for a single LB on evaporated island films. The chemical images created with the SERS/SERRS data provide a mapping of the enhancement factor distribution on the surface coated with the probe molecule. In addition to the simple mapping illustrated in this review, the statistical analysis of the large data collected is under development for single molecule identification, and many potential applications in biology, medicine and materials science. Future developments of LB-SERS or LB-SERRS will harness either the strong signal in the "average" SERS/SERRS experiments or the unique properties of the spectra of isolated species when the statistical average is broken down. In addition, the LB technique may help the development of SERS substrates as one of the methods used in nanolithography.

\section{ACKNOWLEDGEMENTS}

This work was supported by FAPESP, CNPq, Capes, INEO and nBioNet (Brazil). 


\section{REFERENCES}

1. D. L. Feldheim, C. A. Foss, eds. Metal Nanoparticles. Synthesis, Characterization and Applications. Marcel Dekker, Inc., New York (2002).

2. C. S. R. Kumar, J. Hormes, C. Leuschner, eds. Nanofabrication towards biomedical applications. Verlag GmbH \&Co. KGaA, Weinheim (2005).

3. G. Schmid, ed. Nanoparticles. From theory to Applications. WileyVCH Verlag, Essen (2005).

4. J. M. Campelo, D. Luna, R. Luque, J. M. Marinas, A. A. Romero. Chemsuschem 2, 18-45 (2009).

5. N. G. Khlebtsov, L. A. Dykman. Journal of Quantitative Spectroscopy \& Radiative Transfer 111, 1-35 (2010).

6. S. Q. Liu, Z. Y. Tang. Journal of Materials Chemistry 20, 24-35 (2010).

7. M. J. Natan, L. A. Lyon. Metal Nanoparticles: Synthesis, Characterization and Applications, New York (2002).

8. M. G. Albrecht, J. A. Creighton. Journal of the American Chemical Society 99, 5215-5217 (1977).

9. D. L. Jeanmaire, R. P. VanDuyne. Journal of Electroanalytical Chemistry 84, 1-20 (1977).

10. R. Aroca. Surface-enhanced Vibrational Spectroscopy. John Wiley \& Sons, Chichester (2006).

11. M. Fleischmann, P. J. Hendra, A. J. McQuillan. Chemical Physics Letters 26, 163-166 (1974).

12. M. Moskovits. Reviews of Modern Physics 57, 783-826 (1985).

13. K. A. Willets, R. P. Van Duyne. Annual Review of Physical Chemistry 58, 267-297 (2007)

14. E. C. Le Ru, P. G. Etchegoin. Principles of Surface Enhanced Raman Spectroscopy (and related plasmonic effects). Elsevier, Amsterdam (2009.).

15. C. Noguez. Journal of Physical Chemistry C 111, 3806-3819 (2007).

16. H. A. Atwater. Scientific American 296, 56-63 (2007).

17. A. Campion, P. Kambhampati. Chemical Society Reviews 27, 241-250 (1998).

18. A. Otto, I. Mrozek, H. Grabhorn, W. Akemann. Condens. Matter 4, 1143-1212 (1992).

19. K. Kneipp, H. Kneipp. Applied Spectroscopy 60, 322A-334A (2006).

20. C. J. L. Constantino, T. Lemma, P. A. Antunes, R. Aroca. Analytical Chemistry 73, 3674-3678 (2001).

21. K. Kneipp, Y. Wang, H. Kneipp, L. T. Perelman, I. Itzkan, R. R. Dasari, M. S. Feld. Physical Review Letters 78, 1667-1670 (1997).

22. S. Nie, S. R. Emory. Science (Washington, D. C.) 275, 1102-1106 (1997).

23. B. Tolaieb, C. J. L. Constantino, R. F. Aroca. Analyst (Cambridge, United Kingdom) 129, 337-341 (2004).

24. E. J. Bjerneld, Z. Foeldes-Papp, M. Kaell, R. Rigler. Journal of Physical Chemistry B 106, 1213-1218 (2002).

25. P. J. G. Goulet, R. F. Aroca. Analytical Chemistry (Washington, DC, United States) 79, 2728-2734 (2007).

26. R. C. Maher, M. Dalley, E. C. Le Ru, L. F. Cohen, P. G. Etchegoin, H. Hartigan, R. J. C. Brown, M. J. T. Milton. Journal of Chemical Physics $121,8901-8910$ (2004).

27. N. P. W. Pieczonka, R. F. Aroca. Chemical Society Reviews 37, 946954 (2008).

28. R. Zenobi. Chimia 53, 35-37 (1999).

29. R. F. Aroca, R. A. Alvarez-Puebla, N. Pieczonka, S. Sanchez-Cortez, J. V. Garcia-Ramos. Advances in Colloid and Interface Science 116, 4561 (2005).

30. G. A. Baker, D. S. Moore. Analytical and Bioanalytical Chemistry 382, 1751-1770 (2005).

31. G. Braun, I. Pavel, A. R. Morrill, D. S. Seferos, G. C. Bazan, N. O. Reich, M. Moskovits. Journal of the American Chemical Society 129, 7760-7761 (2007).

32. R. F. Aroca, C. J. L. Constantino. Langmuir 16, 5425-5429 (2000).

33. S. B. Chaney, S. Shanmukh, R. A. Dluhy, Y. P. Zhao. Appl. Phys. Lett. 87, 031908-031910. (2005).

34. S. J. Lee, A. R. Morrill, M. Moskovits. Journal of the American Chemical Society 128, 2200-2201 (2006).

35. Y. L. Wang, H. J. Chen, E. K. Wang. Nanotechnology 19, article number105604. (2008).

36. R. F. Aroca, P. J. G. Goulet, D. S. Dos Santos, Jr., R. A. Alvarez-Puebla, O. N. Oliveira, Jr. Analytical Chemistry 77, 378-382 (2005).
37. K. Yliniemi, M. Vahvaselka, Y. V. Ingelgem, K. Baert, B. P. Wilson, H. Terryn, K. Kontturi. J. Mater. Chem. 18, 199-206. (2008).

38. M. Fan, A. G. Brolo. Phys. Chem. Chem. Phys. 11, 7381-7389. (2009).

39. H. Ko, V. V. Tsukruk. Small 4, 1980-1984 (2008).

40. M. Yang, T. Chen, W. S. Lau, Y. Wang, Q. Tang, Y. Yang, H. Chen. Small 5, 198-202 (2009).

41. D. K. Lim, K. S. Jeon, H. M. Kim, J. M. Nam, Y. D. Suh. Nature Materials 9, 60-67 (2010).

42. P. H. B. Aoki, P. Alessio, J. A. De Saja, C. J. L. Constantino. Journal of Raman Spectroscopy 41, 40-48. (2010).

43. X.-M. Lin, Y. Cui, Y.-H. Xu, B. Ren, Z.-Q. Tian. Analytical and Bioanalytical Chemistry 394, 1729-1745 (2009).

44. H. Seki. Journal of Electron Spectroscopy and Related Phenomena 39, 289-310 (1986).

45. L. Rodriguez-Lorenzo, R. A. Alvarez-Puebla, I. Pastoriza-Santos, S. Mazzucco, O. Stephan, M. Kociak, L. M. Liz-Marzan, F. J. G. de Abajo. J. Am. Chem. Soc. 131, 4616-4618. (2009).

46. M. C. Petty. Langmuir-Blodgett Films: An Introduction. Cambridge University Press, Cambridge (1996).

47. G. Roberts. Langmuir-Blodgett Films. Plenum Press, New York (1990).

48. D. A. Long. The Raman Effect. John Wiley \& Sons, Ltd, Chichester (2001).

49. J. J. Laserna. Modern Techniques in Raman Spectroscopy. John Wiley \& Sons, Toronto (1996)

50. E. C. Le Ru, P. G. Etchegoin. Chemical Physics Letters 423, 63-66 (2006).

51. J. P. Camden, J. A. Dieringer, J. Zhao, R. P. Van Duyne. Accounts of Chemical Research 41, 1653-1661 (2008).

52. G. C. Schatz, R. P. Van Duyne. In Handbook of Vibrational Spectroscopy (J. M. C. a. P. R. Griffiths, ed.), pp. 759-774. John Wiley \& Sons, Ltd (2002)

53. K. L. Kelly, E. Coronado, L. L. Zhao, G. C. Schatz. Journal of Physical Chemistry B 107, 668-677 (2003).

54. J. Kneipp, H. Kneipp, K. Kneipp. Chemical Society Reviews 37, $1052-$ 1060 (2008).

55. E. Giorgetti, G. Margheri, S. Sottini, M. Muniz-Miranda. Synthetic Metals 139, 929-932 (2003)

56. G. J. Kovacs, R. O. Loutfy, P. S. Vincett, C. Jennings, R. Aroca. Langmuir 2, 689-694 (1986).

57. G. Mie. Physik. Z. 8, 769 (1908).

58. H. Xu, M. Kall. Sensors and Actuators, B: Chemical B87, 244-249 (2002).

59. H. Xu, X.-H. Wang, M. P. Persson, H. Q. Xu, M. Kall, P. Johansson Physical Review Letters 93, 243002/243001-243002/243004 (2004).

60. W. H. P. Pernice, F. P. Payne, D. F. G. Gallagher. Optics Express 15 , 11433-11443 (2007).

61. M. A. Yurkin, A. G. Hoekstra. Journal of Quantitative Spectroscopy \& Radiative Transfer 106, 558-589 (2007).

62. E. Prodan, C. Radloff, N. J. Halas, P. Nordlander. Science 302, 419-422 (2003).

63. J. Cipriani, S. Racine, R. Dupeyrat, H. Hasmonay, M. Dupeyrat, Y. Levy, C. Imbert. Optics Communications 11 70-73 (1974).

64. R. Aroca, C. Jennings, G. J. Kovac, R. O. Loutfy, P. S. Vincett. Journal of Physical Chemistry 89, 4051-4054 (1985).

65. Y. J. Chen, G. M. Carter, S. K. Tripathy. Solid State Comm. 54, 19-22 (1985).

66. Y. J. Chen, S. K. Tripathy, G. M. Carter, B. S. Elman, E. S. Koteles, J. George. Solid State Comm. 58, 97 (1986).

67. T. M. Cotton, R. A. Uphaus, D. Mobius. J. Phys. Chem. 90, 6071 (1986).

68. D. Battisti, R. Aroca. Journal of Molecular Structure 218, 351-356 (1990).

69. J. H. Kim, T. M. Cotton, R. A. Uphaus, D. Moebius. J. Phys. Chem. 93, 3713-3720 (1989)

70. A. K. Maiti, R. Aroca, Y. Nagao. Journal of Raman Spectroscopy 24, 351-356 (1993).

71. J. Souto, R. Aroca, J. A. DeSaja. Journal of Raman Spectroscopy 22, 349-353 (1991).

72. C. J. L. Constantino, P. A. Antunes, E. C. Venancio, N. Consolin, F. J. Fonseca, L. H. C. Mattoso, R. F. Aroca, O. N. Oliveira, Jr., A. Riul, Jr. Sensor Letters 2, 95-101 (2004).

73. C. J. L. Constantino, R. F. Aroca. Journal of Raman Spectroscopy 31, $887-890$ (2000). 
74. C. J. L. Constantino, R. F. Aroca, J. A. He, V. Zucolotto, L. Li, O. N Oliveira, Jr., J. Kumar, S. K. Tripathy. Applied Spectroscopy 56, $187-$ 191 (2002).

75. C. J. L. Constantino, R. F. Aroca, S. Yang, V. Zucolotto, L. Li, O. N. Oliveira, Jr., A. L. Cholli, J. Kumar, S. K. Tripathy. Journal of Macromolecular Science, Pure and Applied Chemistry A38, 1549-1557 (2001)

76. C. Heywang, M. S.-P. Chazalet, M. Masson, J. Bolard. Spectrosc. Biol. Mol.: New Dir., Eur. Conf., 8th, 339-342 (1999).

77. U. Guhathakurta-Ghosh, R. Aroca, R. O. Loutfy, Y. Nagao. Journal of Raman Spectroscopy 20, 795-800 (1989).

78. J. Huang, C. Li, Y. Liang. Langmuir 16, 3937-3940 (2000).

79. C. Li, J. Huang, Y. Liang. Langmuir 16, $7701-7707$ (2000).

80. R. E. Clavijo, D. Battisti, R. Aroca, G. J. Kovacs, C. A. Jennings Langmuir 8, 113-117 (1992).

81. Y. Gorbunova, M. L. Rodriguez-Mendez, J. Souto, L. Tomilova, J. A. de Saja. Chem. Mater. 7, 1443-1447 (1995).

82. D. Battisti, R. Aroca. Journal of the American Chemical Society 114, 1201-1204 (1992).

83. P. H. B. Aoki, P. Alessio, M. L. Rodriguez-Mendez, J. A. D. Saez, C. J. L. Constantino. Langmuir 25, 13062-13070 (2009).

84. L. Gaffo, C. J. L. Constantino, W. C. Moreira, R. F. Aroca, O. N. Oliveira. Jr. Spectrochimica Acta, Part A: Molecular and Biomolecular Spectroscopy 60A, 321-327 (2004).

85. D. Battisti, L. Tomilova, R. Aroca. Chemistry of Materials 4, 1323 1328 (1992).

86. T. Lemma, R. F. Aroca. Journal of Raman Spectroscopy 33, 197-201 (2002).

87. G. G. Siu, L. Yulong, X. Shishen, X. Jingmei, L. Tiankai, X. Linge. Thin Solid Films 274, 147-149 (1996).

88. M. Ferreira, C. J. L. Constantino, C. A. Olivati, A. L. Vega, D. T. Balogh, R. F. Aroca, R. M. Faria, O. N. Oliveira. Jr. Langmuir 19, 8835 8842 (2003).

89. M. Haro, D. J. Ross, L. Oriol, I. Gascon, P. Cea, M. C. Lopez, R. F. Aroca. Langmuir 23, 1804-1809 (2007).

90. D. Volpati, A. E. Job, R. F. Aroca, C. J. L. Constantino. Journal of Physical Chemistry B 112, 3894-3902 (2008).

91. B. Berno, A. Nazri, R. Aroca. Journal of Raman Spectroscopy 27, 41-47 (1996).

92. E. Johnson, R. Aroca, Y. Nagao. Journal of Physical Chemistry 95, 8840-8843 (1991).

93. A. Feofanov, A. Ianoul, E. Kryukov, S. Maskevich, G. Vasiliuk, L. Kivach, I. Nabiev. Analytical Chemistry 69, 3731-3740 (1997).

94. J. Zhang, J. Zhao, H. X. He, H. L. Zhang, H. L. Li, Z. F. Liu. Langmuir 14, 5521-5525 (1998)

95. M. A. Mahmoud, C. E. Tabor, M. A. El-Sayed. Journal of Physical Chemistry C 113 (14), 5493-5501 (2009).

96. M. A. Mahmoud, M. A. El-Sayed. Nano Letters 9 (8), 3025-3031 (2009).

97. E. Johnson, R. Aroca, J. Pahapill. Journal of Molecular Structure 293, 331-335 (1993)

98. P. J. G. Goulet, N. P. W. Pieczonka, R. F. Aroca. Analytical Chemistry 75, 1918-1923 (2003).

99. P. J. G. Goulet, N. P. W. Pieczonka, R. F. Aroca. Journal of Raman Spectroscopy 36, 574-580 (2005).

100. C. J. L. Constantino, T. Lemma, P. A. Antunes, R. Aroca. Spectrochimica Acta, Part A: Molecular and Biomolecular Spectroscopy 58A, 403-409 (2002).

101. M. Saint-Pierre Chazalet, M. Masson, C. Bousquet, G. Bolbach, Y. Ridente, J. Bolard. Thin Solid Films 244 852-856 (1994).

102. W. Miao, X. Du, Y. Liang. Langmuir 19 (13), 5389-5396 (2003).

103. G. G. Siu, Y. Liu, S. Xie, J. Xu, T. Li, L. Xu. Thin Solid Films $274147-$ 149 (1996).

104. G. Xu, F. Yan. Spectrochimica Acta Part A: Molecular and Biomolecular Spectroscopy 70, 104-108 (2008).

105. C. Heywang, M. S.-P. Chazalet, M. Masson, A. Garnier-Suillerot, J. Bolard. Langmuir 12, 6459-6467 (1996).

106. C. Heywang, M. S.-P. Chazalet, M. Masson, J. Bolard. Biophysical Journal 75, 2368-2381 (1998).
107. C. J. L. Constantino, R. F. Aroca, C. R. Mendonca, S. V. Mello, D. T. Balogh, O. N. Oliveira. Jr. Spectrochimica Acta, Part A: Molecular and Biomolecular Spectroscopy 57A, 281-289 (2001).

108. C. J. L. Constantino, R. F. Aroca, C. R. Mendonca, S. V. Mello, D. T. Balogh, S. C. Zilio, O. N. De Oliveira, Jr. Advanced Functional Materials 11, 65-68 (2001).

109. N. Marquestaut, A. Martin, D. Talaga, L. Servant, S. Ravaine, S. Reculusa, D. M. Bassani, E. Gillies, F. Lagugne-Labarthet. Langmuir 24, 11313-11321 (2008)

110. X. L. Hou, L. X. Wu, W. Q. Xu, L. D. Qin, C. S. Wang, X. Zhang, J. C. Shen. Colloid Surf. A-Physicochem. Eng. Asp. 198, 135-140 (2002).

111. A. Tao, F. Kim, C. Hess, J. Goldberger, R. He, Y. Sun, Y. Xia, P. Yang. Nano Letters 3, 1229-1233 (2003).

112. N. P. W. Pieczonka, G. Moula, R. F. Aroca. Langmuir 25, 11261-11264 (2009).

113. W. E. Moerner. 106, 910-927 (2002)

114. J. R. Lakowicz, C. D. Geddes, I. Gryczynski, J. Malicka, Z. Gryczynski, K. Aslan, J. Lukomska, E. Matveeva, J. Zhang, R. Badugu, J. Huang. Journal of Fluorescence 14, 425-441 (2004).

115. P. G. Etchegoin, M. Meyer, E. Blackie, E. C. Le Ru. Analytical Chemistry ACS ASAP (2007)

116. P. G. Etchegoin, P. D. Lacharmoise, E. C. Le Ru. Analytical Chemistry 81, 682-688 (2009)

117. P. Goulet, N. Pieczonka, R. Aroca. Canadian Journal of Analytical Sciences and Spectroscopy 48, 146-152 (2003).

118. P. J. G. Goulet, R. F. Aroca. Canadian Journal of Analytical Sciences and Spectroscopy 52, 172-177 (2007).

119. K. Kneipp. Experimentelle Technik der Physik (Berlin) 36, 161-166 (1988).

120. K. Kneipp, Y. Wang, R. R. Dasari, M. S. Feld. Applied Spectroscopy 49, 780-784 (1995)

121. K. Kneipp, H. Kneipp, I. Itzkan, R. R. Dasari, M. S. Feld. Chemical Reviews (Washington, D. C.) 99, 2957-2975 (1999).

122. G. T. Taylor, S. K. Sharma, K. Mohanan. Applied Spectroscopy 44, 635-640 (1990).

123. C. Rodger, W. E. Smith, G. Dent, M. Edmondson. Journal of the Chemical Society, Dalton Transactions: Inorganic Chemistry, 791-799 (1996).

124. D. Zeisel, V. Deckert, R. Zenobi, T. Vo-Dinh. Chemical Physics Letters 283, 381-385 (1998).

125. H. Xu, E. J. Bjerneld, M. Kall, L. Borjesson. Physical Review Letters $83,4357-4360$ (1999).

126. C. J. L. Constantino, J. Duff, R. Aroca. Spectroc. Acta Pt. A-Molec. Biomolec. Spectr. 57, 1249-1259 (2001).

127. T.-W. Koo, S. Chan, A. A. Berlin. Optics Letters 30, 1024-1026 (2005).

128. I. Delfino, A. R. Bizzarri, S. Cannistraro. Biophysical Chemistry 113 , 41-51 (2005).

129. A. K. Kalkan, S. J. Fonash. Applied Physics Letters 89, 233103/233101233103/233103 (2006).

130. Z. L. Zhang, Y. F. Yin, J. W. Jiang, Y. J. Mo. Journal of Molecular Structure 920 297-300. (2009).

131. E. J. Blackie, E. C. Le Ru, P. G. Etchegoin. J. Am. Chem. Soc. 131, 14466-14472 (2009).

132. E. C. Le Ru, P. G. Etchegoin, M. Meyer. Journal of Chemical Physics 125, 204701/204701-204701/204713 (2006).

133. S. Nie, S. R. Emory. Science 275, 1102-1106 (1997).

134. W. E. Moerner. J. Phys. Chem. B 106, 910-927 (2002).

135. C. J. Constantino, T. Lemma, P. A. Antunes, R. Aroca. Analytical chemistry 73, 3674-3678. (2001)

136. P. J. G. Goulet, R. F. Aroca. Analytical Chemistry 79, 2728-2734 (2007).

137. E. C. Le Ru, M. Meyer, P. G. Etchegoin. Journal of Physical Chemistry B 110, 1944-1948 (2006)

138. J. A. Dieringer, R. B. Lettan, II, K. A. Scheidt, R. P. Van Duyne. Journal of the American Chemical Society 129, 16249-16256 (2007).

139. P. G. Etchegoin, E. C. Le Ru, M. Meyer. Journal of the American Chemical Society 131, 2713-2716 (2009). 